\title{
CPDeLearning oे
}

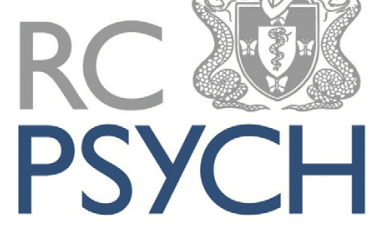

ROYAL COLLEGE OF PSYCHIATRISTS

RCPsych's online learning resource for mental health professionals
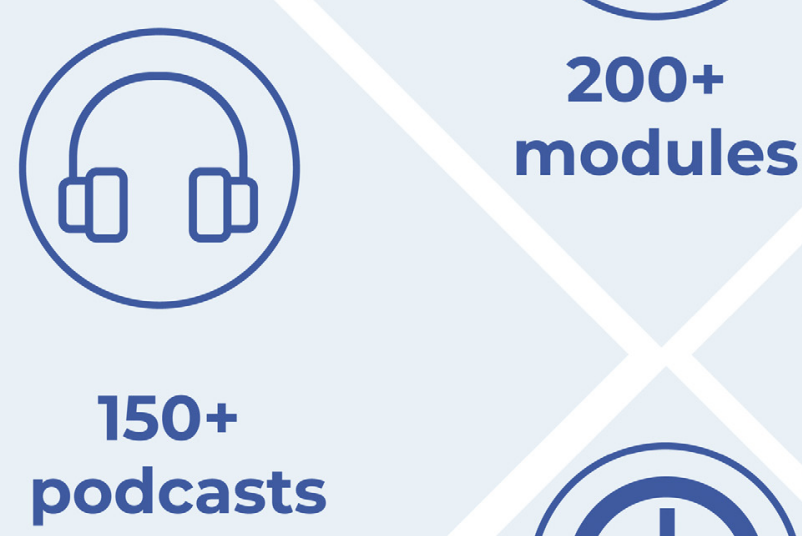
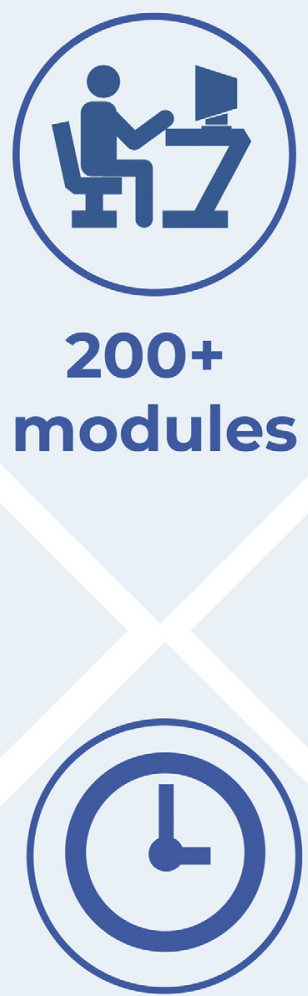

Up to 50 CPD HOURS during the COVID-19 pandemic

\section{Rop?}

Institutional subscriptions

Many workplaces now purchase institutional subscriptions to CPD eLearning, with OpenAthens authentication available. Could you have access through your institution, at no cost to you?

\section{https://elearninghub.rcpsych.ac.uk/}




\section{BJPsych}

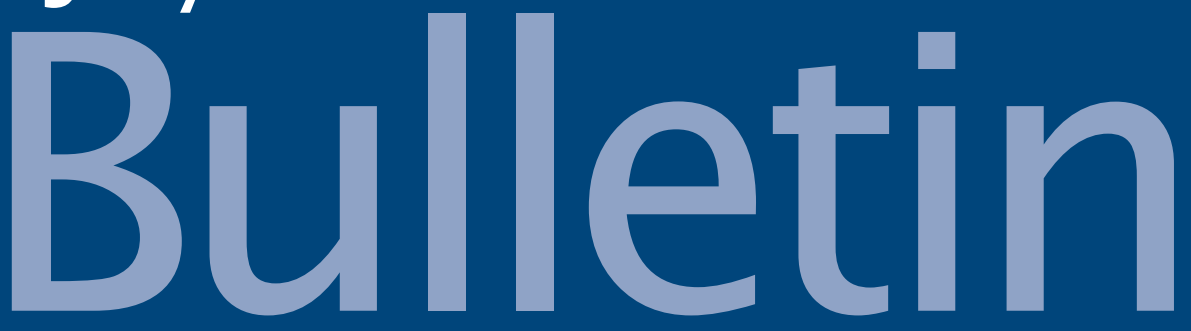

\section{EDITORIAL}

1 Self-harm and suicide in adults: will safety plans keep people safe after self-harm?

Allan House

\section{ORIGINAL PAPERS}

4 Improving attitudes toward electroconvulsive therapy Oakley Cheung, Marc Baker and Paul Tabraham

10 Individual placement and support: cross-sectional study of equality of access and outcome for Black, Asian and minority ethnic communities Rachel Perkins, Rash Patel, Amelia Willett, Laura Chisholm and Miles Rinaldi

\section{CULTURAL REFLECTIONS}

16 Magical thinking and moral injury: exclusion culture in psychiatry

Chloe Beale

\section{SPECIAL ARTICLES}

20 No wrong door: addressing injustices and achieving better mental healthcare provision for under-18s in acute physical healthcare settings Virginia Davies

26 Review of antipsychotic prescribing at HMP/YOI Low Newton Lois Carey and Stephen Barlow

33 Virtual psychiatric care fast-tracked: reflections inspired by the COVID-19 pandemic

Tea Rosic, Sandra Lubert and Zainab Samaan

36 The contagiousness of memes: containing the spread of COVID-19 conspiracy theories in a forensic psychiatric hospital

Reena Panchal and Alexander Jack
42 Effects of decision aids for depression treatment in adults: systematic review

Christoper A. Alarcon-Ruiz, Jessica Hanae Zafra-Tanaka, Mario E. Diaz-Barrera, Naysha Becerra-Chauca,

Carlos J. Toro-Huamanchumo, Josmel Pacheco-Mendoza, Alvaro Taype-Rondan and Jhony A. De La Cruz-Vargas

\section{PRAXIS}

52 Transforming MRCPsych theory examinations: digitisation and very short answer questions (VSAQs)

Karl Scheeres, Niruj Agrawal, Stephanie Ewen and Ian Hall

\section{EDUCATION AND TRAINING}

57 Transference-focused psychotherapy as an aid to learning psychodynamic psychotherapy: qualitative analysis of UK psychiatry trainees' views

Orestis Kanter Bax, Georgios Nerantzis and Tennyson Lee

\section{COLUMNS}

64 Interview with Dr Jan Wise

Abdi Sanati

\section{Correspondence}

71 Obituary

Vivienne Cohen

\section{Erratum}

74 In Memoriam

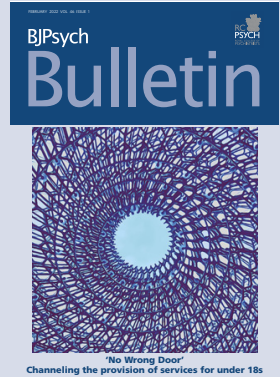

This issue contains an article by Virginia Davies (pp. 20-26) about the distressing reality of the provision of mental healthcare for children and young people in acute trusts settings. The article provides arguments for a better integration of mental healthcare into physical healthcare settings, as well as tasks and challenges for commissioners. 